\title{
Influencia de la duración en el reconocimiento de las modalidades oracionales en el español colombiano*
}

\author{
Influence of Duration on the Recognition of \\ Sentence Modes in Colombian Spanish
}

Mercedes Amparo Muñetón Ayala**

\section{RESUMEN}

Este trabajo analizó la influencia de las configuraciones durativas en la diferenciación de las modalidades oracionales en la producción y percepción del habla. En el estudio de producción participaron 36 hablantes de seis de las principales ciudades de Colombia. Las diferencias se ubicaron en el sintagma preposicional, donde la configuración L* caracteriza las oraciones declarativas y la B* las interrogativas. Para verificar este resultado a nivel perceptivo, 42 estudiantes universitarios de diferentes partes del país escucharon oraciones sintetizadas en las que el sintagma preposicional de las declarativas contenía la L* y el de las interrogativas la $\mathrm{B}^{*}$. Los participantes diferenciaron acertadamente las modalidades oracionales. Los resultados sugieren que la estructura temporal del habla diferencia las modalidades oracionales en la producción y en la percepción.

\section{ABSTRACT \\ This research paper analyzed the influence of the configuration of vowel duration to differentiate sentence modality at the level of production and perception. Thirty-six speakers from six Co- lombian cities participated in the production study. Differences were found in the prepositional syntagma (PS), where the $\mathrm{L}^{*}$ con-}

Palabras clave: configuraciones durativas, prosodia, diferencias mínimas perceptibles.

Keywords: configuration of vowel duration, prosody, just noticeable difference.

El trabajo se realizó en el marco del proyecto AMPER_Col, integrado al grupo de investigación "Psicolingüística y prosodia", coordinado por la profesora Mercedes Muñetón y perteneciente a la Universidad de Antioquia.

Colombiana. Doctora en psicolingüística experimental de la Universidad de La Laguna. Profesora Titular de la Universidad de Antioquia, Medellín, Colombia. mercedes. muneton@udea.edu.co 
figuration characterizes the declarative while the $\mathrm{B}^{*}$ is associated with interrogative sentences. To verify the results at a perceptive level, 42 university students listened to synthesized sentences where the PS of the declaratives contained the $\mathrm{L}^{*}$ and the interrogatives the $\mathrm{B}^{*}$ configuration. Participants correctly differentiated these sentence modalities. The overall results suggest that the temporal structure of speech allows us to differentiate sentence modalities both at the level of production and perception. 


\section{Introducción}

La prosodia es un rasgo complejo de la producción oral que proporciona melodía al habla. Su complejidad radica en la confluencia de tres parámetros acústicos: la frecuencia fundamental (F0), la duración y la amplitud. De ellos, el más estudiado ha sido la frecuencia fundamental, porque se ha señalado como el parámetro que marca la prominencia en el habla (Kochanski, Grabe, Coleman \& Rosner, 2005). De esta manera, los otros dos parámetros se han dejado un poco de lado, aunque diferentes estudios han mostrado su importancia no solo en español (Canellada y Madsen, 1987; Cuervo, 1954; Navarro Tomas, 1944), sino también en inglés (Fry, 1995; Turk \& Sawusch, 1996). Así, Canellada y Madsen (1987) sugieren que el correlato del acento es la duración, mientras que Cuervo (1954) y Navarro Tomas (1963) señalan que es la intensidad. Además, estudios llevados a cabo en lengua inglesa indican que la duración y la intensidad son claves para juzgar el acento, sin embargo, la duración es un parámetro más efectivo que la amplitud (Fry, 1995; Turk \& Sawusch, 1996).

Fox (2000) menciona que, de todos los rasgos prosódicos, la longitud parece ser la más simple y la menos controversial. La producción verbal se lleva a cabo en tiempo real y, a diferencia de los segmentos del habla - que suelen superponerse en algunas ocasiones-, siempre hay un orden temporal tanto para la producción como para la percepción del habla. Así, cualquier parte de esta señal ocupará una porción definida de tiempo - la cual puede ser medida-, y la duración de estas partes son solo el tiempo tomado para ser producidas oralmente. Por tanto, el habla es una actividad dependiente del tiempo que podría influir, incluso, a nivel modal. Definitivamente, la mayor o menor duración vocálica se evidencia solamente a través del contraste entre los sonidos (Fox, 2000). Por tal motivo, con el fin de aprehender sus diferencias tanto a nivel intra como intersujeto se hace necesario contrastar los segmentos para generar unidades relativas que sean comparables. Actualmente, gracias a la profundización en el análisis de la duración, se ha observado que este parámetro puede ser un rasgo que permita diferenciar las modalidades oracionales (Muñetón y Dorta, 2019; Muñiz Cachón, 2017), aunque como es bien sabido, esta es una característica atribuida solamente a la F0. 
En esta línea, Muñiz Cachón (2017), en el marco del proyecto AMPER-Astur ${ }^{1}$, mostró la influencia que ejerce la duración en la discriminación de las modalidades oracionales a nivel de producción oral. El estudio fue realizado con las voces de un hombre y una mujer hablantes de castellano y otros tantos hablantes de asturiano, todos sin estudios superiores, nacidos y residentes en Mieres, localidad del centro sur de Asturias. Concretamente, los autores atribuyen la diferencia modal al incremento de duración de las oraciones interrogativas en comparación con las declarativas, en el límite de frontera final de oraciones con expansión en el sujeto y en el objeto.

Además, en estudios previos en el marco del proyecto AMPER-Col ${ }^{2}$ (Muñetón y Dorta, 2019; Muñetón, Díaz y Dorta, 2018) se ha generado la propuesta de las configuraciones durativas, las cuales permiten extraer la estructura temporal que subyace al español hablado en diferentes localidades, mediante la comparación tripartita de las tónicas con sus adyacentes. Las etiquetas básicas son larga (L) y breve (B), las cuales se marcan con un asterisco cuando son tónicas y así es posible establecer las configuraciones de estas con las pretónicas y postónicas. Para ello, hemos usado la propuesta del umbral mínimo perceptivo definido por Pamies Bertrán y Fernández Planas (2006) en un 33,33\%. Por tanto, se considera que hay diferencias significativas entre dos vocales cuando la duración de una supera a la otra en el umbral mínimo sugerido.

Los estudios de las configuraciones durativas han mostrado que existen básicamente ocho estructuras:

1) L La tónica es significativamente más larga que la pretónica y postónica.

2) $B^{*} \quad$ No hay diferencias significativas entre la pretónica-tónica, ni entre tónica-postónica.

AMPER es la sigla del proyecto internacional Atlas Multimedia de Prosodia del Espacio Románico. Uno de los objetivos principales es el estudio de los tres parámetros prosódicos (F0, duración e intensidad) en las distintas lenguas y variedades diatópicas del ámbito románico. La identificación del país en estudio se hace mediante la extensión después de la sigla AMPER, así AMPER-Astur, significa proyecto AMPER en Asturias.

2 AMPER-Col, AMPER en Colombia, está coordinado por Mercedes Amparo Muñetón Ayala, profesora de la Universidad de Antioquia, Medellín, Colombia y subcoordinado por Josefa Dorta Luis, profesora de la Universidad de La Laguna, Tenerife, Islas Canarias, España. 
3) $\mathrm{BL}^{*} \quad$ Solo hay diferencias entre pretónica-tónica, siendo la tónica la más larga.

4) L*B Solo hay diferencias entre tónica-postónica, siendo la tónica la más larga.

5) $\mathrm{LB}^{*}$ Solo hay diferencias entre la pretónica-tónica, siendo la pretónica la más larga.

6) $\mathrm{B}^{*} \mathrm{~L} \quad$ Solo hay diferencias entre la tónica-postónica, siendo la postónica la más larga.

7) BLL La tónica es más larga que la pretónica y, a su vez, la postónica es más larga que la tónica

8) LB* Hay diferencias significativas entre la tónica y las dos adyacentes, siendo aquella breve y estas largas.

Las comparaciones de estas estructuras han evidenciado diferencias y similitudes, por ejemplo, entre Venezuela y Colombia. En estos países vecinos la comparación entre las oraciones declarativas y las interrogativas mostró que la configuración L $\mathrm{L}^{*}$ presenta un alto porcentaje en el sintagma preposional (SP) de ambas modalidades en el habla colombiana, mientras que en Venezuela constituye el segundo porcentaje más alto en el SP, siendo el primero el BL*. Es importante destacar que este estudio se realizó solamente en voz femenina de dos localidades en Venezuela (Caracas y Mérida) y dos en Colombia (Bogotá y Medellín). También se encontró que las tónicas del SP en relación con las tónicas del sintagma nominal (SN) y del sintagma verbal (SV) son más largas, independientemente del país o la modalidad, mostrando que la duración es un parámetro determinante para marcar el tonema, como lo sugieren Fernández Planas y Martínez Celdrán (2003).

Otro estudio llevado a cabo en Colombia por Muñetón y Dorta (2019) con el fin de determinar y describir las configuraciones durativas de oraciones declarativas e interrogativas en un corpus de habla experimental emitido por mujeres y hombres de cinco de las principales ciudades del país (Bogotá, Medellín, Cali, Barranquilla e Ibagué), mostró que la configuración $\mathrm{B}^{*}$ se presenta en las interrogativas con mayor frecuencia que en las declarativas, mientras que en la configuración L* sucede lo contrario, pues las declarativas aventajan a las interrogativas.

Los trabajos anteriores, debido al número de participantes (cuatro en los dos primeros y 10 en el último), usaron la descripción de frecuencias o el análisis del chi cuadrado $(\chi 2)$, que permiten observar si los resulta- 
dos se deben o no al azar. Sin embargo, estos resultados no dan cabida a examinar si la duración interactúa con la modalidad oracional, con los sintagmas, o con ambos factores al tiempo. Además, no se llevaron a cabo estudios perceptivos para validar los resultados obtenidos.

Para responder a estas preguntas, en el presente estudio hemos llevado a cabo un diseño en el que interviene la modalidad, los sintagmas y las configuraciones como variables independientes intrasujeto y la variable dependiente es la producción media de cada configuración. Además, con el fin de comprobar la veracidad perceptiva de los resultados obtenidos en el primer estudio, posteriormente contrastaremos si las diferencias encontradas en la producción son reconocidas a nivel perceptivo. Para ello, aislaremos las configuraciones que diferencian las modalidades oracionales a nivel estadístico y contrastaremos si los oyentes identifican las oraciones como declarativas o interrogativas, teniendo como referencia solo la duración. En resumen, el objetivo de la investigación fue analizar la influencia de las configuraciones durativas en la diferenciación de las modalidades oracionales tanto a nivel de producción como de percepción.

\section{Estudio de producción}

El propósito de la primera parte del estudio fue examinar si las configuraciones durativas intervienen en la diferenciación de las modalidades oracionales a nivel de producción.

\subsection{Método}

\subsubsection{Participantes}

En esta etapa se analizaron las emisiones de 36 colombianos de seis puntos de encuesta: Bogotá, Medellín, Cali, Barranquilla, Ibagué y Pasto. Cada punto estuvo constituido por seis informantes con edades comprendidas entre los 25 y 45 años: un hombre y una mujer sin estudios de la zona rural; un hombre y una mujer sin estudios de la zona urbana; y un hombre y una mujer con estudios de la zona urbana.

\subsubsection{Diseño y material}

El proyecto AMPER consta de cuatro corpus: el fijo o formal, el situacional, el Map Task y la conversación informal (Contini, 2005). En este 
estudio analizamos el corpus fijo, el cual consta de variables controladas en su estructura oracional (oraciones sin expansión, con expansión en el sujeto y con expansión en el objeto) y tipología acentual (palabras oxítonas, paroxítonas y proparoxítonas); todas las combinaciones posibles dan como resultado 63 oraciones en cada modalidad (declarativa e interrogativa). El corpus se presenta a los informantes de forma aleatoria y se graba un mínimo de tres repeticiones que se analizaran posteriormente.

Además, seleccionamos nueve oraciones (tres en cada estructura oracional) para cada modalidad oracional, por tanto, el total por sujeto ascendió a 18. Teniendo en cuenta que de cada oración se presentan tres repeticiones ( 54 oraciones por sujeto) pronunciadas por los 36 informantes, el total asciende a 1.944. El criterio de selección se basó en que el sintagma nominal (con y sin expansión) y el preposicional (con y sin expansión) debían coincidir en su estructura acentual, ya que el sintagma verbal permanece invariable en todas las oraciones. La selección se verificó de esta manera para que no hubiera vocales tónicas juntas. Las oraciones fueron del tipo: la máquina se toca con pánico; el bandolín español se toca con emoción; la guitarra se toca con paciencia finita.

\subsubsection{Procedimiento}

Las grabaciones de los hablantes de Medellín se hicieron en la cabina insonorizada del laboratorio de fonética de la Universidad de Antioquia y las del resto de participantes en su lugar de residencia, utilizando grabadoras TASCAM-100, con micrófono unidireccional externo, modelo Sennheiser E845. Los datos fueron analizados posteriormente con el programa AMPER-2006 (López et al., 2007) en el entorno Matlab Matriz Laboratory ${ }^{3}$. Las rutinas creadas en Matlab permiten obtener, a partir de la segmentación en el oscilograma, tres valores de F0 de cada vocal silábica (en el inicio, en el medio y en el final), así como el valor de la duración y de la intensidad. En este estudio nos enfocamos solo en la duración.

\subsubsection{Codificación de los datos}

La codificación de los datos se llevó a cabo en siete pasos. En primer lugar, se extrajo el valor de la duración de cada uno de los segmen-

Licencia No 878.004 del laboratorio de fonética de la Universidad de Antioquia. 
tos vocálicos de las 1.944 oraciones; a continuación, por cada oración usamos el valor medio de las tres repeticiones pronunciadas por cada informante, por tanto, finalmente contamos con un total de 648 oraciones. De estas hubo 72 oraciones por cada estructura oracional (SE, ES y EO $)^{4}$ y cada tipología acentual $(\mathrm{O}, \mathrm{P}, \mathrm{Pp})^{5} ; 36$ de modalidad declarativa y otro tanto de modalidad interrogativa (ver Tabla 1). En segundo término, se segmentaron las oraciones en tres grupos sintagmáticos SN (sintagma nominal), SV (sintagma verbal) y sintagma preposicional (SP). Las oraciones con expansión en el sujeto y en el objeto constaban de dos constituyentes: el correspondiente al sustantivo y a la expansión. Todo se distribuyó en función de la modalidad.

En la tercera etapa, cada una de las vocales se rotularon como pretónica, tónica y postónica, ya sea por fonética normal o fonética sintáctica. Por ejemplo, si hay que rotular la secuencia "el bandolín se toca" la "e" de la sílaba "se" es la postónica de la sílaba "lín". De esta manera, el total de vocales rotuladas asciende a 6.912. Su conteo es como sigue: en las oraciones SE se contabilizaron tres vocales tónicas y sus adyacentes, lo cual asciende a nueve vocales, excepto en las oxítonas que son solo ocho por obvias razones, dando un total de 1.872 vocales; en las oraciones con ES y con EO se contabilizaron 12 para las paroxítonas y proparoxítonas y 11 para las oxítonas, dando un total de 2.520 vocales para cada una de las estructuras oracionales (Ver Tabla 1).

Tabla 1.

Número de vocales rotuladas en función de la estructura oracional y tipología acentual.

\begin{tabular}{ccccc}
\hline Oraciones & Oxítonas & Paroxítonas & Proparoxítonas & Total vocales \\
\hline SE & $72 \times 8$ & $72 \times 9$ & $72 \times 9$ & 1.872 \\
\hline ES & $72 \times 11$ & $72 \times 12$ & $72 \times 12$ & 2.520 \\
\hline EO & $72 \times 11$ & $72 \times 12$ & $72 \times 12$ & 2.520 \\
\hline & & Total vocales & 6.912 \\
\hline
\end{tabular}

Nota. Las abreviaturas de la tabla son: SE = Sin expansión; ES = Expansión en el sujeto; EO = Expansión en el objeto.

Fuente: Elaboración propia.

$4 \quad$ Abreviaturas de la estructura oracional: $\mathrm{SE}=$ Sin Expansión; $\mathrm{ES}=$ Expansión en el sujeto; y EO = Expansión en el objeto.

5 Abreviaturas de la tipología acentual: $\mathrm{O}=$ Oxítona; $\mathrm{P}=$ Paroxítona; y $\mathrm{Pp}=$ Proparoxítona. 
En el cuarto paso se generaron las DMP entre cada contraste de la tónica con su adyacente: pretónica-tónica y tónica-postónica. En la elaboración de los contrastes se tuvo en cuenta su tipología oracional debido a que el contraste tónica-postónica es inexistente al final del SP de las oxítonas. Así, por ejemplo, en las oraciones SE oxítonas se generan 216 contrastes en Pr-T, debido a que hay tres tónicas en cada una de las 72 oraciones; pero en el contraste T-Ps encontramos 144, porque solo hay dos tónicas en las cuales podemos llevar a cabo la comparación. En total, se generaron 4.536 contrastes: 2.376 en Pr-T y 2.160 en T-Ps (ver Tabla 2).

Tabla 2.

Total de contrastes en función de la estructura oracional y tipología oracional.

\begin{tabular}{ccccccccccccccc}
\hline & \multicolumn{1}{c}{ Oxítonas } & \multicolumn{1}{c}{ Paroxítonas } & \multicolumn{2}{c}{ Proparoxítonas } \\
\hline & \multicolumn{1}{c}{ Pr-T } & T-Ps & \multicolumn{2}{c}{ Pr-T } & T-Ps & Pr-T & T-Ps & \multicolumn{2}{c}{ Total } \\
\hline & T & C & T & C & T & C & T & C & T & C & T & C & Pr-T & T-Ps \\
\hline SE & 3 & 216 & 2 & 144 & 3 & 216 & 3 & 216 & 3 & 216 & 3 & 216 & 648 & 576 \\
\hline ES & 4 & 288 & 3 & 216 & 4 & 288 & 4 & 288 & 4 & 288 & 4 & 288 & 864 & 792 \\
\hline EO & 4 & 288 & 3 & 216 & 4 & 288 & 4 & 288 & 4 & 288 & 4 & 288 & 864 & 792 \\
\hline & & & & & & & & & & & & Total & 2.376 & 2.160 \\
\hline
\end{tabular}

Nota. Las abreviaturas de la tabla son: $\mathrm{T}$ = Tónica; $\mathrm{C}=$ Contraste; $\mathrm{Pr}=$ Pretónica; $\mathrm{Ps}=$ Postónica.

Fuente: Elaboración propia.

En el quinto paso, se crearon las configuraciones durativas, las cuales ascienden a 2.376 debido a que por una tónica y sus adyacentes hay una configuración. Por tanto, el número de vocales tónicas corresponde al número de configuraciones. En el sexto paso se estableció una base de datos en la cual hubo tres factores: la modalidad, los sintagmas y las configuraciones durativas, siendo la variable dependiente la media de producción de cada configuración, razón por la cual el valor de la producción de una configuración solo podía ser uno o cero. En el séptimo paso se usó análisis multivariado de medidas repetidas de los tres factores.

\subsection{Resultados}

Presentaremos los resultados en tres grandes apartados: en el primero observaremos el comportamiento de la duración basado en las medias 
generales en función de los sintagmas oracionales y su modalidad; en el segundo veremos la distribución de las configuraciones durativas; y en el tercero observaremos el análisis multivariado de las configuraciones en relación con las modalidades oracionales y sus sintagmas en cada una de las estructuras oracionales.

\subsubsection{Análisis de las medias generales}

En este apartado se llevó a cabo una serie de pruebas $t$ para los contrastes de muestras independientes entre los sintagmas, teniendo en cuenta la modalidad y la estructura oracional.

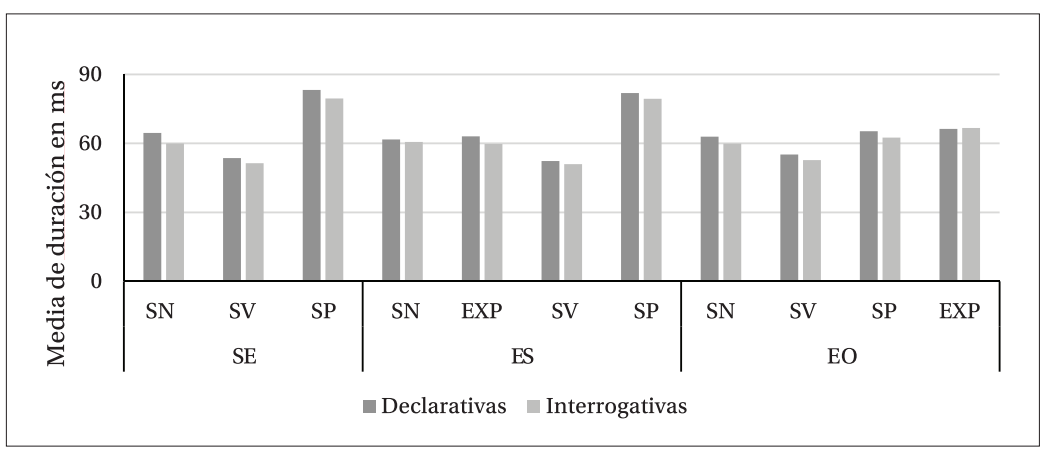

Figura 1. Media en ms de los sintagmas en función de la estructura oracional y de la modalidad.

Nota. Abreviaturas usadas: $\mathrm{SE}$ = Sin expansión; ES = Expansión en el sujeto; EO = Expansión en el objeto; SN = Sintagma nominal; SV = Sintagma verbal; SP = Sintagma preposicional; y EXP = Expansión.

Fuente: Elaboración propia.

Observando los datos en conjunto, encontramos que, independientemente de la modalidad o la estructura oracional, la duración tiende a ser mayor en el SP que en el SN, tanto en las oraciones declarativas (SE: $\mathrm{t}(35)=-9,06, p<, 000$; ES: $\mathrm{t}(35)=-10,71, p<, 000$; EO: $\mathrm{t}(35)=-2,09, p<, 043)$; como en las interrogativas (SE $\mathrm{t}(35)=-12,88$, $p<, 000$; ES t(35) = -9,04, $p<, 000$; EO t(35) $=-4,56, p<, 000)$, como lo muestran los análisis por pares. Por otra parte, la Tabla 3 indica que las oraciones declarativas tienen una duración significativamente mayor que las interrogativas en cada sintagma, excepto en el SN de las oraciones con ES, y en las EXP de las oraciones con EO en donde no se encuentran diferencias. 
Tabla 3.

Media, desviación estándar y efectos significativos (P) al contrastar cada sintagma en función de la modalidad oracional.

\begin{tabular}{cccccccc}
\hline & & \multicolumn{3}{c}{ Declarativas } & \multicolumn{3}{c}{ Interrogativas } \\
\cline { 2 - 8 } & & N & Media & DE & Media & DE & P \\
\hline \multirow{3}{*}{ SE } & SN & 36 & 64,55 & 11,38 & 59,94 & 10,76 & 0,000 \\
\cline { 2 - 8 } & SV & 36 & 53,63 & 7,36 & 51,40 & 7,14 & 0,000 \\
\cline { 2 - 8 } & SP & 36 & 83,25 & 13,69 & 79,55 & 12,88 & 0,003 \\
\hline \multirow{3}{*}{ ES } & SN & 36 & 61,73 & 10,98 & 60,67 & 12,51 & 0,223 \\
\cline { 2 - 8 } & EXP & 36 & 63,08 & 12,24 & 59,74 & 11,09 & 0,001 \\
\cline { 2 - 8 } & SV & 36 & 52,31 & 7,39 & 50,98 & 6,70 & 0,038 \\
\cline { 2 - 8 } & SP & 36 & 81,89 & 10,46 & 79,39 & 12,74 & 0,033 \\
\hline \multirow{2}{*}{ EO } & SN & 36 & 62,94 & 12,06 & 59,92 & 11,31 & 0,001 \\
\cline { 2 - 8 } & SV & 36 & 55,18 & 8,17 & 52,75 & 7,88 & 0,001 \\
\cline { 2 - 8 } & SP & 36 & 65,31 & 8,81 & 62,53 & 9,27 & 0,001 \\
\cline { 2 - 8 } & EXP & 36 & 66,34 & 9,54 & 66,73 & 11,53 & 0,702 \\
\hline
\end{tabular}

Fuente: Elaboración propia.

\subsubsection{Análisis de las configuraciones}

En este apartado se presenta la distribución de las configuraciones observadas en el corpus colombiano tanto en las oraciones declarativas como interrogativas teniendo en cuenta su estructura oracional y sintagmática.

Tabla 4.

Distribución de las configuraciones en función de la modalidad, estructura oracional y sintagmas.

\begin{tabular}{|c|c|c|c|c|c|c|c|c|c|c|c|c|c|}
\hline \multicolumn{14}{|c|}{ Declarativas } \\
\hline \multicolumn{5}{|c|}{ SE } & \multicolumn{2}{|c|}{ ES } & \multicolumn{5}{|c|}{ EO } & \multirow[b]{2}{*}{ Total } & \multirow[b]{2}{*}{$\%$} \\
\hline CONF. & SN & SV & SP & SN & EX & SV & SP & SN & SV & SP & EX & & \\
\hline $\mathrm{B}^{*}$ & 37 & 85 & 16 & 47 & 49 & 83 & 21 & 47 & 79 & 52 & 42 & 558 & 23,48 \\
\hline $\mathrm{B}^{*} \mathrm{~L}$ & 0 & 5 & 0 & 2 & 1 & 4 & 1 & 0 & 5 & 0 & 0 & 18 & 0,76 \\
\hline $\mathrm{BL}^{*}$ & 11 & 17 & 50 & 12 & 1 & 19 & 48 & 10 & 20 & 9 & 25 & 222 & 9,34 \\
\hline $\mathrm{BL}^{*} \mathrm{~L}$ & 0 & 0 & 0 & 1 & 0 & 0 & 0 & 0 & 0 & 0 & 0 & 1 & 0,04 \\
\hline $\mathrm{L}^{*}$ & 20 & 1 & 37 & 14 & 10 & 0 & 31 & 16 & 2 & 21 & 31 & 183 & 7,70 \\
\hline $\mathrm{L}^{*} \mathrm{~B}$ & 40 & 0 & 5 & 32 & 46 & 0 & 7 & 35 & 1 & 26 & 10 & 202 & 8,50 \\
\hline
\end{tabular}




\begin{tabular}{cccccccccccccc}
\hline $\mathrm{LB}^{*}$ & 0 & 0 & 0 & 0 & 1 & 1 & 0 & 0 & 1 & 0 & 0 & 3 & 0,13 \\
\hline $\mathrm{LB}^{*} \mathrm{~L}$ & 0 & 0 & 0 & 0 & 0 & 1 & 0 & 0 & 0 & 0 & 0 & 1 & 0,04 \\
\hline \multicolumn{10}{c}{ Interrogativas } \\
\hline $\mathrm{B}^{*}$ & 40 & 78 & 32 & 56 & 54 & 85 & 34 & 47 & 81 & 61 & 42 & 610 & 25,67 \\
\hline $\mathrm{B}^{*} \mathrm{~L}$ & 0 & 4 & 0 & 1 & 1 & 3 & 1 & 0 & 6 & 0 & 1 & 17 & 0,72 \\
\hline $\mathrm{BL}$ & 8 & 23 & 54 & 9 & 2 & 17 & 52 & 11 & 19 & 7 & 38 & 240 & 10,10 \\
\hline $\mathrm{L}^{*}$ & 19 & 2 & 18 & 7 & 7 & 1 & 15 & 11 & 1 & 11 & 19 & 111 & 4,67 \\
\hline $\mathrm{L}^{*} \mathrm{~B}$ & 41 & 0 & 4 & 35 & 43 & 0 & 6 & 38 & 0 & 29 & 8 & 204 & 8,59 \\
\hline $\mathrm{LB}^{*}$ & 0 & 0 & 0 & 0 & 1 & 1 & 0 & 1 & 0 & 0 & 0 & 3 & 0,13 \\
\hline $\mathrm{LB}^{*} \mathrm{~L}$ & 0 & 1 & 0 & 0 & 0 & 1 & 0 & 0 & 1 & 0 & 0 & 3 & 0,13 \\
\hline
\end{tabular}

Fuente: Elaboración propia.

La Tabla 4 muestra que el corpus en estudio generó las ocho configuraciones que han sido reseñadas en Muñetón y Dorta (2019) y en Muñetón et al. (2018) De estas, en el 50,84\% intervienen DMP, con un porcentaje ligeramente superior para las declarativas que para las interrogativas (26,51\% y 24,33\%, respectivamente). Además, estos resultados coinciden en señalar que la producción de $\mathrm{B}^{*} \mathrm{~L}, \mathrm{LB}^{*}, \mathrm{LB}{ }^{*} \mathrm{~L}$ y $\mathrm{BL} \mathrm{L}^{*} \mathrm{~L}$ ( $1,93 \%$ en total) es incipiente en comparación con el corpus en general, por tanto, de cara a los análisis estadísticos vamos a considerar las cuatro configuraciones con mayor representatividad, esto es, $\mathrm{B}^{*}, \mathrm{~L}^{*} \mathrm{~B}$, $\mathrm{BL}^{*}$ y L* $(98,07 \%$ en total $)$.

\subsubsection{Análisis multivariado}

La Tabla 5 muestra las medias y desviaciones estándar de la producción de las configuraciones durativas en función de la modalidad y el sintagma en cada una de las estructuras oracionales. De esta forma, se llevó a cabo un análisis multivariado de medidas repetidas de tres factores para examinar el efecto de la modalidad (D/I) ${ }^{6} \mathrm{x}$ sintagma (SN/ $\mathrm{SV} / \mathrm{SP} / \mathrm{EXP}) \mathrm{x}$ configuraciones $\left(\mathrm{B}^{*} / \mathrm{BL}^{*} / \mathrm{L}^{*} / \mathrm{L}^{*} \mathrm{~B}\right)$ en cada una de las tipologías oracionales. En este diseño se comprueba si existen diferencias entre las modalidades en función de los sintagmas y las configuraciones en cada una de las estructuras oracionales.

Abreviaturas: D = Declarativa; I =Interrogativa. 
Tabla 5.

Medias y desviaciones típicas de la producción de las configuraciones durativas en función de la modalidad y el sintagma en cada una de las estructuras oracionales.

\begin{tabular}{|c|c|c|c|c|c|c|c|c|}
\hline \multirow[b]{2}{*}{ Modalidad } & \multirow[b]{2}{*}{ Sintagma } & \multirow[b]{2}{*}{ Configuración } & \multicolumn{2}{|c|}{$\begin{array}{c}\text { Sin } \\
\text { expansión }\end{array}$} & \multicolumn{2}{|c|}{$\begin{array}{c}\text { Expansión } \\
\text { sujeto }\end{array}$} & \multicolumn{2}{|c|}{$\begin{array}{c}\text { Expansión } \\
\text { objeto }\end{array}$} \\
\hline & & & Media & DT & Media & DT & Media & DT \\
\hline \multirow[t]{16}{*}{ Declarativa } & $\mathrm{SN}$ & $\mathrm{B}^{*}$ & 0,34 & 0,05 & 0,44 & 0,04 & 0,44 & 0,04 \\
\hline & & $\mathrm{BL}^{*}$ & 0,10 & 0,03 & 0,11 & 0,03 & 0,09 & 0,03 \\
\hline & & $\mathrm{L}^{*}$ & 0,19 & 0,04 & 0,13 & 0,03 & 0,15 & 0,03 \\
\hline & & $\mathrm{L}^{*} \mathrm{~B}$ & 0,37 & 0,05 & 0,30 & 0,04 & 0,32 & 0,04 \\
\hline & SV & $\mathrm{B}^{*}$ & 0,79 & 0,05 & 0,77 & 0,05 & 0,73 & 0,05 \\
\hline & & $\mathrm{BL}^{*}$ & 0,16 & 0,05 & 0,18 & 0,05 & 0,19 & 0,05 \\
\hline & & $\mathrm{L}^{*}$ & 0,01 & 0,01 & 0,00 & 0,00 & 0,02 & 0,01 \\
\hline & & $\mathrm{L}^{*} \mathrm{~B}$ & 0,00 & 0,00 & 0,00 & 0,00 & 0,01 & 0,01 \\
\hline & SP & $\mathrm{B}^{*}$ & 0,15 & 0,04 & 0,19 & 0,04 & 0,48 & 0,05 \\
\hline & & $\mathrm{BL}^{*}$ & 0,46 & 0,05 & 0,44 & 0,04 & 0,08 & 0,02 \\
\hline & & $\mathrm{L}^{*}$ & 0,34 & 0,04 & 0,29 & 0,04 & 0,19 & 0,04 \\
\hline & & $\mathrm{L}^{*} \mathrm{~B}$ & 0,05 & 0,02 & 0,06 & 0,02 & 0,24 & 0,04 \\
\hline & EXP & $\mathrm{B}^{*}$ & & & 0,45 & 0,05 & 0,39 & 0,05 \\
\hline & & $\mathrm{BL}^{*}$ & & & 0,01 & 0,01 & 0,23 & 0,04 \\
\hline & & $\mathrm{L}^{*}$ & & & 0,09 & 0,03 & 0,29 & 0,04 \\
\hline & & $\mathrm{L}^{*} \mathrm{~B}$ & & & 0,43 & 0,04 & 0,09 & 0,03 \\
\hline \multirow[t]{16}{*}{ Interrogativa } & $\mathrm{SN}$ & $\mathrm{B}^{*}$ & 0,37 & 0,05 & 0,52 & 0,04 & 0,44 & 0,05 \\
\hline & & $\mathrm{BL}^{*}$ & 0,07 & 0,02 & 0,08 & 0,02 & 0,10 & 0,03 \\
\hline & & $\mathrm{L}^{*}$ & 0,18 & 0,03 & 0,06 & 0,02 & 0,10 & 0,03 \\
\hline & & $L^{*} B$ & 0,38 & 0,04 & 0,32 & 0,04 & 0,35 & 0,04 \\
\hline & SV & $\mathrm{B}^{*}$ & 0,72 & 0,05 & 0,79 & 0,05 & 0,75 & 0,06 \\
\hline & & $\mathrm{BL}^{*}$ & 0,21 & 0,05 & 0,16 & 0,05 & 0,18 & 0,05 \\
\hline & & $\mathrm{L}^{*}$ & 0,02 & 0,02 & 0,01 & 0,01 & 0,01 & 0,01 \\
\hline & & $\mathrm{L}^{*} \mathrm{~B}$ & 0,00 & 0,00 & 0,00 & 0,00 & 0,00 & 0,00 \\
\hline & SP & $\mathrm{B}^{*}$ & 0,30 & 0,05 & 0,31 & 0,05 & 0,56 & 0,05 \\
\hline & & $\mathrm{BL}^{*}$ & 0,50 & 0,05 & 0,48 & 0,04 & 0,06 & 0,03 \\
\hline & & $\mathrm{L}^{*}$ & 0,17 & 0,03 & 0,14 & 0,03 & 0,10 & 0,03 \\
\hline & & $\mathrm{L}^{*} \mathrm{~B}$ & 0,04 & 0,02 & 0,06 & 0,02 & 0,27 & 0,04 \\
\hline & EXP & $\mathrm{B}^{*}$ & & & 0,50 & 0,05 & 0,39 & 0,05 \\
\hline & & $\mathrm{BL}^{*}$ & & & 0,02 & 0,01 & 0,35 & 0,04 \\
\hline & & $\mathrm{L}^{*}$ & & & 0,06 & 0,03 & 0,18 & 0,03 \\
\hline & & $L^{*} B$ & & & 0,40 & 0,04 & 0,07 & 0,02 \\
\hline
\end{tabular}

Fuente: Elaboración propia. 


\section{a) Oraciones sin expansión}

Los análisis mostraron un efecto principal de configuraciones $\mathrm{F}(3,33)$ $=46,75, p<, 000$, la interacción modalidad $\mathrm{x}$ configuraciones $\mathrm{F}(3,33)$ $=3,20, p<, 036$ y sintagma x configuraciones $\mathrm{F}(3,33)=36,77, p<, 000$ fueron significativas, como también lo fue la interacción triple, $\mathrm{F}(6,30)$ $=36,77, p<, 000$. Con el fin de explicar la interacción de más alto nivel, los datos se organizaron en dos figuras basadas en la modalidad. La Figura 1 muestra los resultados en los diferentes sintagmas por cada una de las configuraciones en la modalidad declarativa, en tanto que la Figura 2 muestra los mismos datos, pero en la modalidad interrogativa.

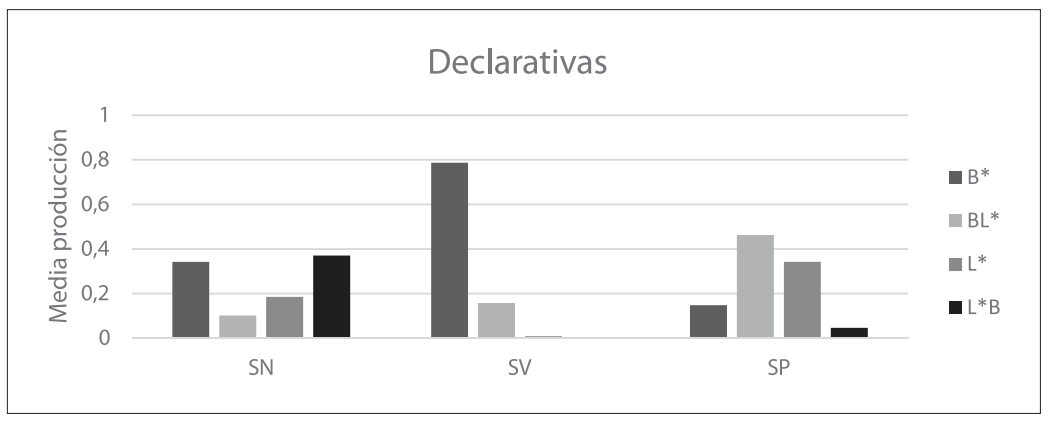

Figura 1. Media de producción de las configuraciones en los diferentes sintagmas de las oraciones sin expansión en la modalidad declarativa.

Fuente: Elaboración propia.

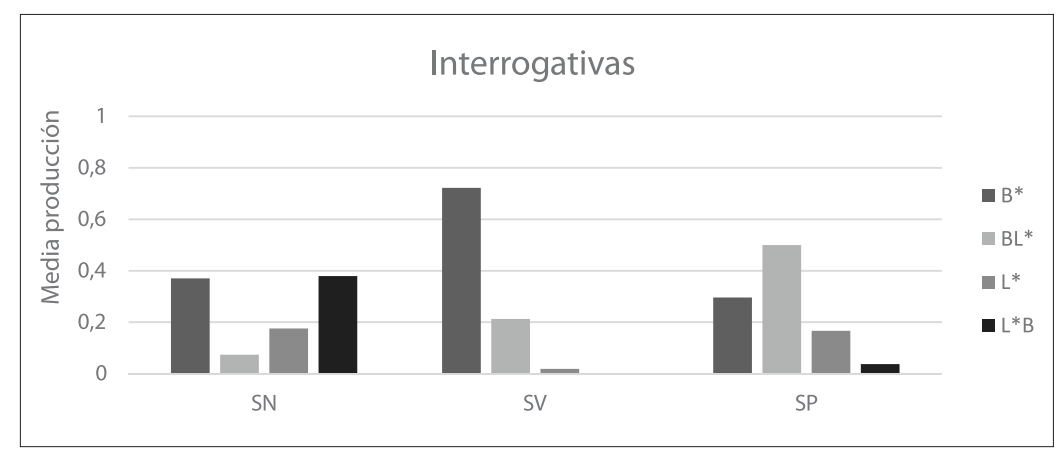

Figura 2. Media de producción de las configuraciones en los diferentes sintagmas de las oraciones sin expansión en la modalidad interrogativa. Fuente: Elaboración propia.

Las Figuras 1 y 2 muestran que la producción media de cada configuración es similar en ambas modalidades, sin embargo, podemos 
observar las diferencias en el SP, donde $\mathrm{B}^{*}$ presenta una mayor producción en las interrogativas que en las declarativas $[\mathrm{t}(35)=-3,29, p<$ ,002)], mientras que L* presenta una mayor producción en las declarativas que en las interrogativas $[\mathrm{t}(35)=4,84, p<, 000)]$. Este contraste es interesante debido a que $\mathrm{B}^{*}$ significa ausencia de diferencias, mientras que $L^{*}$ significa que la tónica es significativamente más larga que sus adyacentes.

\section{b) Oraciones con expansión en el sujeto}

Los análisis mostraron un efecto principal de configuraciones $\mathrm{F}(3$, $33)=44,34, p<, 000$, las interacciones dobles, modalidad $\mathrm{x}$ configuraciones $\mathrm{F}(3,33)=10,31, p<, 000 \mathrm{y}$ sintagma $\mathrm{x}$ configuraciones $\mathrm{F}(9,27)=36,77, p<, 000$, fueron significativas, como también lo fue la interacción triple, $\mathrm{F}(9,27)=36,77, p<, 004$. Como en la anterior estructura, los datos se organizaron en dos figuras basadas en la modalidad. La Figura 3 y la Figura 4 muestran los resultados en la modalidad declarativa e interrogativa, respectivamente, en los diferentes sintagmas por cada una de las configuraciones. Los resultados puntualizan el mismo patrón descrito anteriormente, esto es, $\mathrm{B}^{*}$ presenta una mayor producción en las interrogativas que en las declarativas $[\mathrm{t}(35)=-2,84, p<, 007)]$, mientras que $\mathrm{L}^{*}$ presenta una mayor producción en las declarativas que en las interrogativas [t(35) $=4,09, p<, 000)]$. Como podemos observar ambas estructuras oracionales presentan un modelo muy claro: la ausencia de diferencias marca el sintagma interrogativo, mientras que la presencia marca el sintagma declarativo.

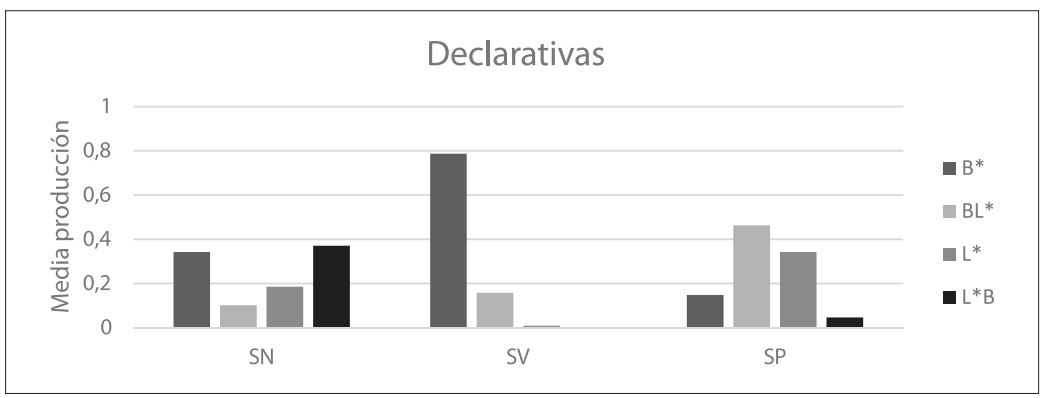

Figura 3. Media de producción de las configuraciones en los diferentes sintagmas de las oraciones con expansión en el sujeto en la modalidad declarativa. Fuente: Elaboración propia. 


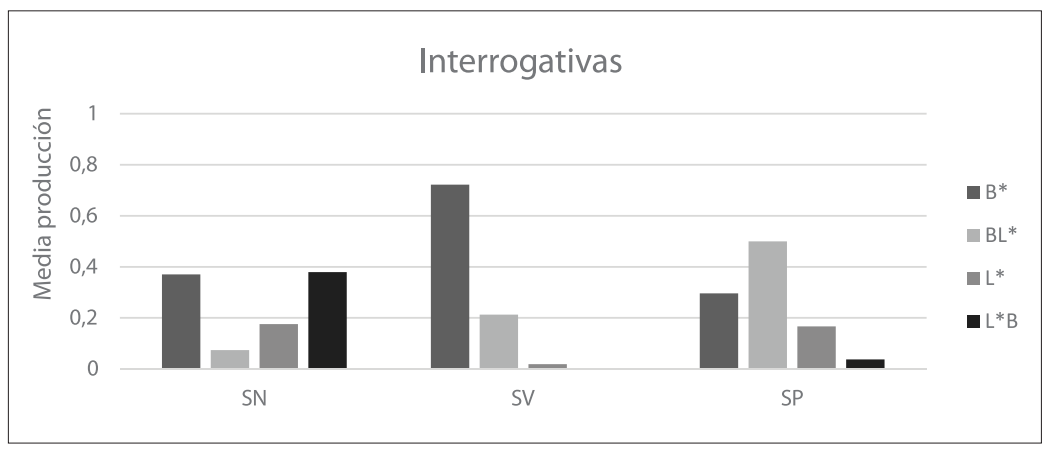

Figura 4. Media de producción de las configuraciones en los diferentes sintagmas de las oraciones con expansión en el sujeto en la modalidad interrogativa. Fuente: Elaboración propia.

\section{c) Oraciones con expansión en el objeto}

En esta estructura oracional encontramos un efecto principal de configuraciones $\mathrm{F}(3,33)=33,80, p<, 000$, mediatizada por una interacción doble entre modalidad y configuraciones $\mathrm{F}(3,33)=5,27, p<, 004$.

La Figura 5 muestra que la producción media de las configuraciones es similar en ambas modalidades, sin embargo, podemos observar las diferencias en la configuración $\mathrm{L}^{*}$, y aquí se vuelve a repetir el patrón encontrado en las dos estructuras oracionales anteriores: la producción de esta configuración es significativamente mayor en las declarativas que en las interrogativas $[\mathrm{t}(35)=4,15, p<, 000)]$.

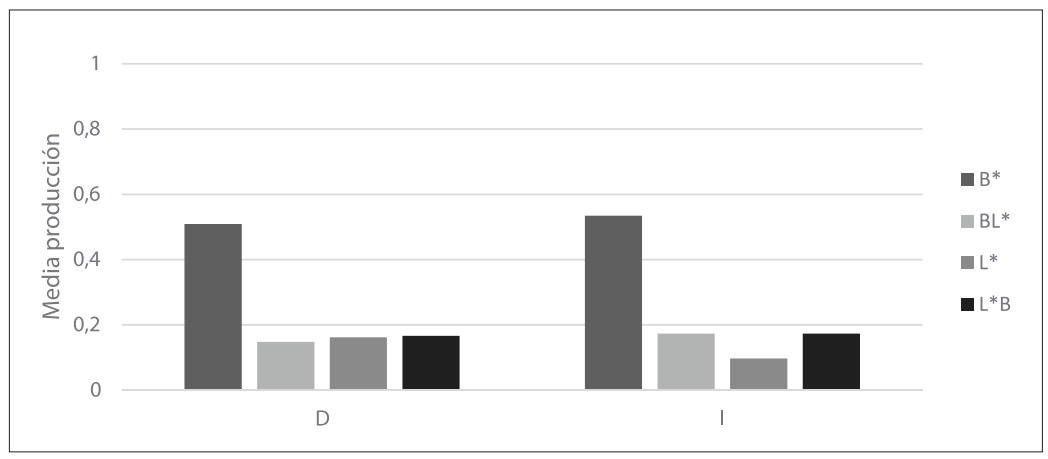

Figura 5. Media de producción de las configuraciones en función de las modalidades oracionales.

Fuente: Elaboración propia. 
Si bien tenemos claro que no hubo una interacción triple, con el fin de profundizar un poco más en los datos llevamos a cabo análisis $t$-test para medidas repetidas, contrastando cada configuración de cada sintagma entre las modalidades. Los datos nos muestran que hay diferencias significativas entre las declarativas y las interrogativas solamente en el SP $[\mathrm{t}(35)=-2,84, p<, 031)]$ y la EXP $[\mathrm{t}(35)=2,64, p<, 012)]$ en la configuración $\mathrm{L}^{*}$, siendo mayor la producción en las declarativas que en las interrogativas. Este dato está en línea con lo hallado en las otras dos estructuras.

\section{Test de percepción}

Con el fin de comprobar la veracidad perceptiva de los resultados obtenidos llevamos a cabo una segunda parte del estudio a través de un test de percepción, aislando las configuraciones que diferencian ambas modalidades.

\subsection{Método}

\subsubsection{Participantes}

En este experimento participaron 45 estudiantes universitarios (23 mujeres y 22 hombres, con una media de edad de 21 años, en un rango que abarcó entre los 18 y los 25 años) de diferentes localidades de Colombia. Todos los participantes eran neurológicamente sanos, colombianos hablantes nativos del español, escribían con la mano derecha y no tenían problemas de audición. Tres participantes se descartaron debido a que no comprendieron la tarea, dejando una muestra final de 42 personas, de las cuales 22 eran mujeres.

\subsubsection{Diseño y material}

De las oraciones recopiladas en el primer estudio se seleccionaron dos oraciones sin expansión que cumplieran el criterio de las declarativas y dos que cumplieran el criterio de las interrogativas. Esto es, en el SP de las primeras, la vocal tónica debía ser significativamente más larga que las adyacentes como se muestra en la Figura 6 y en las otras, la vocal tónica no debía presentar diferencias con sus adyacentes (ver Figura 7). Por tal motivo, se tomaron oraciones con tipología acentual paroxítona. A continuación, se extrajo su síntesis y se quitó su contenido léxico. El test se diseñó usando el software E-prime 3(Psychology 
software tools). Se registraron los aciertos y la latencia de la respuesta. Este último dato se refiere al intervalo transcurrido entre la presentación del estímulo y el inicio de la respuesta. Su relevancia radica en que permite observar la complejidad del procesamiento cognitivo en el reconocimiento de la modalidad. Según De Vega (1992) entre más complejo es el proceso mental, mayor será la latencia invertida en este.

La frecuencia fundamental de las oraciones se manipuló para que fuera similar en toda la oración, con el objetivo de que no influenciara la selección de los participantes. De igual manera, se eliminaron los picos en la amplitud, para lo cual se usó el software PRAAT (Boersma \& Weenink, 2018).

\subsubsection{Procedimiento}

Durante el experimento los participantes se sentaron en una silla cómoda y usaron auriculares. Se les indicó que iban a escuchar oraciones sintetizadas, es decir, la melodía de la oración sin el contenido léxico. Se les pidió que indicaran si la oración que escuchaban era declarativa o interrogativa. Cada participante fue instruido para que respondiera lo más rápido posible una vez que la había escuchado: si era declarativa debía de pulsar la tecla "S" y si era interrogativa, la tecla "L". Al participante se le alertaba de la emisión del próximo ítem mediante la presentación de un asterisco en el centro de la pantalla que duraba $1.000 \mathrm{~ms}$.

Después de recibir las instrucciones los participantes escucharon ocho oraciones de práctica, seguidas por un bloque experimental constituido por 20 ensayos presentados al azar, 10 pertenecientes a las oraciones declarativas y otros tantos a las interrogativas. Cada ensayo consistía en presentar auditivamente una oración. 


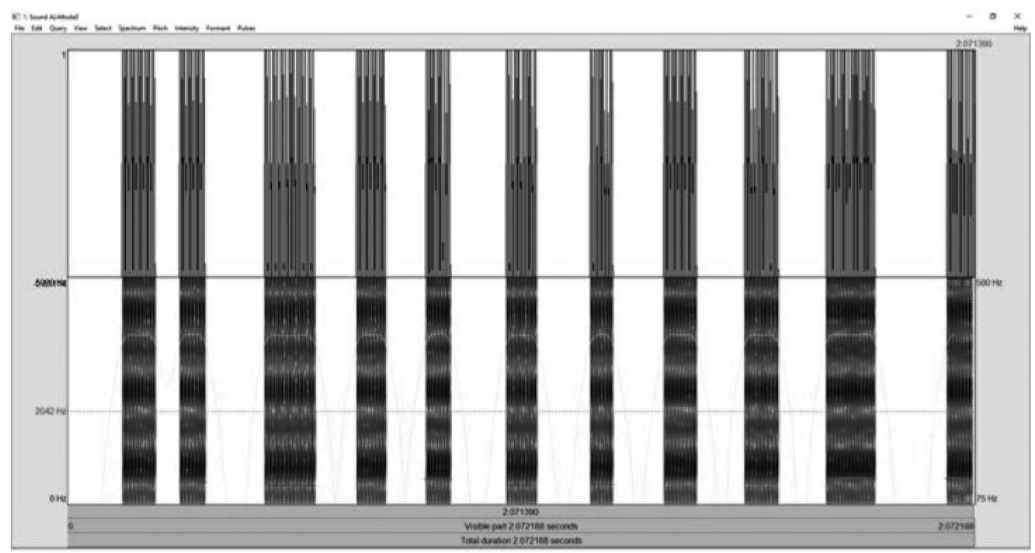

Figura 6. Espectro de oración declarativa. Las barras negras muestran la duración de las vocales tónicas.

Fuente: Elaboración propia.

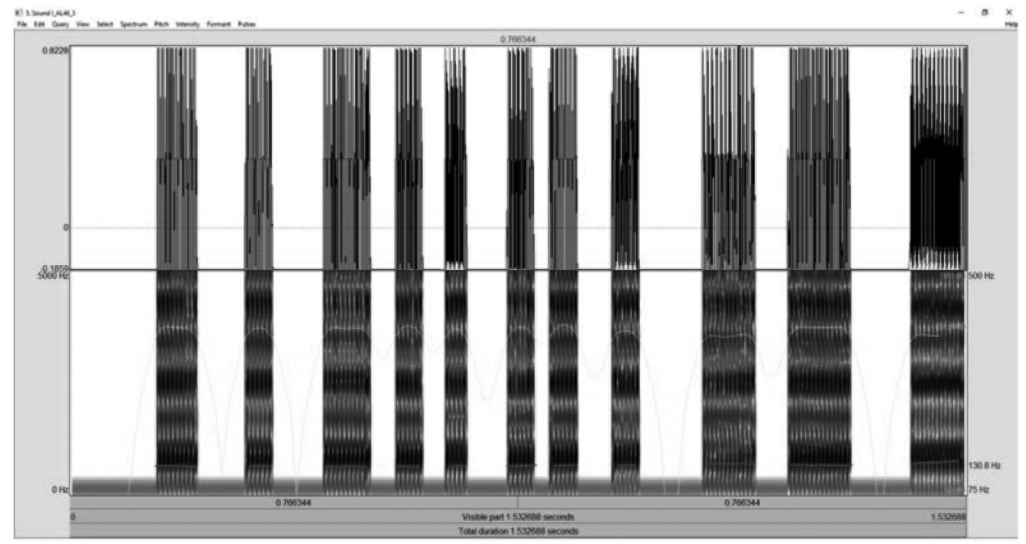

Figura 7. Espectro de oración interrogativa. Las barras negras muestran la duración de las vocales tónicas. La línea azul señala la manipulación de la F0 y la amarilla la amplitud.

Fuente: Elaboración propia.

\subsection{Resultados}

El objetivo de la segunda parte del estudio fue analizar si la duración de las vocales del SP de las oraciones mediatiza el reconocimiento de las modalidades oracionales como se mostró en los resultados 
del estudio de producción. Para ello, comparamos las frecuencias de aciertos y errores mediante el estadístico descriptivo del chi cuadrado $(\chi 2)$, porque nos permite observar si la distribución entre las variables podría o no ser atribuida al azar. Los datos generales, es decir, sin diferenciar entre declarativas e interrogativas, mostraron que la frecuencia de los aciertos en comparación con la de los errores está por encima del azar $(\chi 2=73,70, p<0,000)$. Al realizar el mismo análisis en cada modalidad encontramos un patrón similar en las declarativas $(\chi 2=102,06, p<0,000)$ y en las interrogativas $(\chi 2=$ $4,16, p<0,041)$. Por tanto, podemos inferir que la duración de las vocales en posición final de la oración ayuda a la discriminación entre las modalidades.

A continuación, comparamos las modalidades oracionales mediante un $t$-test de medidas repetidas, tomando como variables dependientes los aciertos y la latencia. Los resultados mostraron que los participantes aciertan más en las oraciones declarativas que en las interrogativas $(t(41)=3,90 ; p<0,000)$; sin embargo, la latencia es inferior en las interrogativas que en las declarativas $(t(41)=4,39 ; p$ $<0,000$ ). Ello parece sugerir que el participante detecta más rápido una oración interrogativa, mientras que en las declarativas les resulta más complejo.

Tabla 6.

Media y DT en la modalidad declarativa e interrogativa en función de los aciertos y la latencia de la respuesta.

\begin{tabular}{cccc}
\hline & & Declarativas & Interrogativas \\
\hline Aciertos & M & 0,67 & 0,53 \\
\hline Latencia & DT & 0,21 & 0,10 \\
\hline & M & 3029,62 & 2906,79 \\
\hline
\end{tabular}

Fuente: Elaboración propia.

\section{Discusión}

El objetivo de la investigación aquí descrita fue analizar la influencia de las configuraciones durativas en la diferenciación de las modalidades oracionales tanto a nivel de producción como de percepción, para lo cual se llevaron a cabo dos estudios, uno por cada nivel. Los 
participantes fueron diferentes en cada caso. En el primero, se llevó a cabo un análisis general de medias, la descripción de las configuraciones durativas y el análisis estadístico de la producción de las configuraciones, mientras que en el segundo, se realizó un test perceptivo que permitió validar los resultados encontrados en el nivel de la producción oral.

En relación con el primer estudio, el contraste de medias mostró que entre el SN y el SP aparecen diferencias durativas, siendo el último el que presenta una mayor duración independientemente de la modalidad. Resultados similares se mencionan en la voz de hablantes femeninas venezolanas y colombianas (Muñetón et al., 2018) o en el español estándar peninsular (Fernández Planas y Martínez Celdrán, 2003). Sin embargo, Muñiz Cachón (2017) reporta una mayor duración para las vocales del SN que para el SP en las oraciones declarativas, mientras que las interrogativas siguen el patrón mencionado anteriormente, en hablantes de castellano y asturiano de Mieres (Asturias). No obstante, mirando los resultados en conjunto, estos apoyan la importancia de la duración como un rasgo determinante para marcar el tonema, como ha sido sugerido por Fernández Planas y Martínez Celdrán (2003) y, también podría ser un rasgo diferenciador de los diversos modos de habla.

Las configuraciones durativas encontradas en esta investigación son las mismas que han sido reportadas hasta el momento (Muñetón et al., 2018; Muñetón y Dorta, 2019). Los porcentajes de producción de las DMP que intervienen en las configuraciones se producen alrededor del 45\% al 50\%. Por ejemplo, en el estudio de Muñetón y Dorta (2019) se produce en un 45,29\%; en el de Fernández Planas y Martínez Celdrán (2003) lo hace en el $46 \%^{7}$; y en el presente estudio se verifica en el 50,84\%. Por tanto, en términos generales la configuración que tiene una mayor frecuencia es aquella que no presenta diferencias entre la tónica y sus adyacentes, esto es, la B*. De igual forma, la distribución de las configuraciones que contienen DMP es similar a la referida en Muñetón y Dorta (2019); así, las configuraciones que acumulan la mayor frecuencia son $\mathrm{BL}^{*}(19,44 \%), \mathrm{L}^{*} \mathrm{~B}(17,09 \%)$ y $\mathrm{L}^{*}$

Dato extraído de la Tabla 9 del estudio de Fernández Planas y Martínez Celdrán (2003). 
(12,37\%). Las otras cuatro configuraciones B* $\mathrm{L}, \mathrm{LB}^{*}, \mathrm{LB}^{*} \mathrm{~L}$ y $\mathrm{BL}{ }^{*} \mathrm{~L}$ ofrecen un porcentaje acumulado incipiente (1,93\% en total). De esta manera, los estudios coinciden en mostrar que las cuatro configuraciones que concentran la mayor frecuencia son $\mathrm{B}^{*}, \mathrm{BL}^{*}, \mathrm{~L}^{*} \mathrm{~B}$ y L*. En relación con la diferenciación de las modalidades en general, Muñetón y Dorta (2019) reportan que la producción de $\mathrm{B}^{*}$ es mayor en las interrogativas que en las declarativas, mientras que estas últimas son caracterizadas por la configuración $\mathrm{L}^{*}$.

Esta tendencia se ratifica en el análisis multivariado de medidas repetidas (modalidad*sintagma*configuraciones) que realizamos en el presente estudio, debido a que la media de producción de estas dos configuraciones es, precisamente, la que mediatiza las diferencias entre las modalidades en función de los diferentes sintagmas. En primer lugar, en las oraciones sin expansión y con expansión en el sujeto se presenta la interacción de los tres factores indicando dependencia entre ellos. Así, ambas tipologías oracionales coinciden en ubicar las diferencias en el SP, donde la producción de la configuración L* es significativamente mayor en las oraciones declarativas que en las interrogativas, mientras que la producción de la $\mathrm{B}^{*}$, es mayor en las interrogativas que en las declarativas. En las oraciones con expansión en el sujeto encontramos una interacción doble entre modalidad y configuraciones, ratificando el patrón encontrado en las otras dos estructuras oracionales, esto es, la configuración L L $^{*}$ presenta una mayor producción en las declarativas que en las interrogativas. Sin embargo, debido al objetivo de la investigación decidimos llevar a cabo un contraste de medias entre cada una de las configuraciones en cada sintagma y en cada modalidad por separado. Los resultados coincidieron en ubicar las diferencias entre las configuraciones solamente en el SP, ya que en el resto de sintagmas no hubo diferencias significativas de forma similar que en las oraciones SE y con ES.

Según lo anterior, el diseño usado nos permite observar que las modalidades oracionales sí se diferencian por la producción de las configuraciones durativas que se presentan específicamente en el SP. Con el fin de contrastar este resultado a nivel perceptivo, llevamos a cabo un test de percepción en donde aislamos las configuraciones reportadas en el SP. Los resultados mostraron que los participantes 
diferenciaban acertadamente las modalidades por encima del azar. Además, también se observó que el nivel de aciertos era superior en las declarativas que en las interrogativas, pero la latencia sigue un patrón contrario, es decir, los oyentes invierten más tiempo en el reconocimiento de las declarativas que en el de las interrogativas. Teniendo en cuenta que la latencia de la respuesta es inversamente proporcional a la activación de la información, esto es, cuanto mayor sea la activación menor será el tiempo de respuesta, los datos en conjunto sugieren que la síntesis de las oraciones activa más rápidamente la información relacionada con la prosodia de las interrogativas que la de las declarativas.

Por último, la investigación aquí descrita proporciona evidencia de la importancia de la duración en la discriminación de las modalidades oracionales tanto a nivel de producción como a nivel perceptivo. Podemos ubicar la diferencia entre las modalidades en el SP, donde las oraciones declarativas son caracterizadas por la configuración L* y las interrogativas por la B*. Estas dos configuraciones difieren porque la tónica en la L* presenta DMP con sus adyacentes a la vez, mientras que en la otra no evidencia ninguna diferencia con sus adyacentes. Sin embargo, consideramos conveniente analizar más adelante hasta qué punto estos resultados se pueden generalizar a otras lenguas románicas.

\section{Referencias bibliográficas}

Boersma, P. \& Weenink, D. (2018). PRAAT: Doing phonetics by computer [Computer program]. Version 6.0.37. Recuperado de http://www.praat.org/

Canellada, M. y Madsen, J. (1987). Pronunciación del español. Lengua hablada y literaria. Madrid: Castalia.

Contini, M. (2005). $2^{\mathrm{e}}$ Séminaire international du projet AMPER, Projet AMPER. Géolinguistique Hors-Série, 3, 1-11.

Cuervo, R. J. (1954). Notas a la gramática de la lengua castellana de don Andrés Bello. Bogotá: Instituto Caro y Cuervo.

De Vega, M. (1992). Introducción a la psicología cognitiva. Madrid: Alianza psicología.

Fernández Planas, A. y Martínez Celdrán, E. (2003). El tono fundamental y la duración: dos aspectos de la taxonomía prosódica en 
dos modalidades de habla (enunciativa e interrogativa) del español. Estudios de Fonética Experimental, 12, 166-200. Recuperado de https://www.raco.cat/index.php/EFE/article/ view/140007

Fox, A. (2000). Prosodic feature and prosodic structure. The phonology of suprasegmentals. Oxford: Oxford University Press.

Fry, D. B. (1995). Duration and intensity as physical correlates of linguistics stress. Journal of the Acoustical Society of America, 27(4), 765-768. https://doi.org/10.1121/1.1908022

Kochanski, G., Grabe, E., Coleman, J., \& Rosner, B. (2005). Loudness predicts prominence: Fundamental frequency lends little. Journal of Acoustical Society of America, 118(2), 1038-1054. https://doi.org/10.1121/1.1923349

López, M., Muñiz, C., Díaz, L., Corral, N., Brezmes, D., y Alvarellos, M. (2007). Análisis y representación de la entonación. Replanteamiento metodológico en el marco del proyecto AMPER. En J. Dorta (Ed.), La prosodia en el ámbito lingüístico románico (pp. 17-34). Madrid/Tenerife: La Página ediciones/Colección Universidad.

Muñetón, M. y Dorta, J. (2019). Estudio de la duración en el marco de la entonación de las principales ciudades de Colombia. Estudios de Fonética Experimental, 28, 161-184. Recuperado de https:/www.raco.cat/index.php/EFE/article/ view/365422/459463

Muñetón, M., Díaz, Ch., y Dorta, J. (2018). La duración en oraciones sin expansión en la voz femenina de dos países fronterizos: Colombia (Bogotá-Medellín) y Venezuela (CaracasMérida). Literatura y Lingüistica, 37, 401-433. https://doi. org/10.29344/0717621x.37.1389

Muñiz Cachón, C. (2017). Implicaciones de la duración en la prosodia: asturiano y castellano del centro de Asturias. Estudios de Fonética Experimental, 14, 223-243. http://stel.ub.edu/labfon/ sites/default/files/XXVI-11-CMuniz.pdf

Navarro Tomás, T. (1944). Manual de entonación española. Madrid: Guadarrama.

Pamies Bertrán, A. y Fernández Planas, M. (2006). La percepción de la duración vocálica en español. Actas del V Congreso Andaluz de Lingüistica General: Homenaje al Profesor José Andrés de Molina Redondo, 1, 501-513. 
Turk, A. E. \& Sawusch, W. (1996). The processing of duration and intensity cues to prominence. Journal of the Acoustical Society of America, 99(6), 3782-3790. https://doi. org/10.1121/1.414995 